\title{
ÓLEOS VOLÁTEIS DE ESPÉCIES DE Myrcia NATIVAS DO RIO GRANDE DO SUL
}

\author{
Renata P. Limberger, Marcos Sobral e Amélia T. Henriques* \\ Faculdade de Farmácia, Universidade Federal do Rio Grande do Sul, Av. Ipiranga 2752, 90610-000 Porto Alegre - RS \\ Chantal Menut e Jean-Marie Bessière \\ Laboratoire de Chimie Oganique-Physique, Université de Montpellier II, Sciences et Techniques du Languedoc 34053 Montpellier \\ Cedex 5, France
}

Recebido em 11/12/03; aceito em 15/6/04; publicado na web em 8/10/04

\begin{abstract}
ESSENTIAL OILS FROM Myrcia SPECIES NATIVE TO RIO GRANDE DO SUL. Essential oils from M. richardiana, $M$. arborescens, M. selloi, M. oligantha, M. rostrata, M. lajeana, M. obtecta, M. pubipetala and M. hatschbachii were obtained by hydrodistillation and analyzed by GC and GC/MS. Sixty-seven compounds have been identified ranging from 90-99\% of the oil contents. All analyzed species were rich in cyclic sesquiterpenes (66-99\%), mainly those from the cadinane, caryophyllane and germacrane cyclization pathway, among them $\beta$-caryophyllene, germacrene $\mathrm{D}$, bicyclogermacrene, $\delta$-cadinene, spathulenol, caryophyllene oxide, globulol and $\alpha$-cadinol. The acyclic sesquiterpene series was well represented by M. lajeana (32.1\%), with $25,3 \%$ of (E)-nerolidyl acetate.
\end{abstract}

Keywords: Myrcia; essential oil; Myrtaceae.

\section{INTRODUÇÃO}

A família Myrtaceae compreende cerca de 140 gêneros, com aproximadamente 3.000 espécies divididas em 2 subfamílias, Myrtoideae e Leptospermoideae ${ }^{1}$. No Brasil, todos os representantes nativos pertencem à subfamília Myrtoideae, a qual é constituída de apenas de uma tribo, Myrteae, que se divide em três subtribos, Eugeniinae, Myrciinae e Myrtinae'. O gênero Myrcia DC. pertence à subtribo Myrciinae e é um dos maiores gêneros americanos de Myrtaceae, com mais de 300 espécies distribuídas do México até o sul do Brasil, sendo 17 dessas espécies encontradas no Rio Grande do $\mathrm{Sul}^{2}$.

Algumas espécies de Myrcia são utilizadas na medicina popular ${ }^{3}$, destacando-se M. multiflora (Lam.) DC. (pedra-ume-caá), utilizada como hipoglicemiante na forma de infuso ou decocto ${ }^{4}$. Estudos farmacológicos demonstraram atividade de extratos de folhas de $M$. fallax (Rich.) DC. frente a células cancerígenas tipo $\mathrm{KB}^{5}$ e atividade antidiabética de mirciatricina I e mirciafenona $\mathrm{B}$, isoladas de folhas de $M$. multiflora, associada à inibição da atividade das enzimas aldose redutase e alfa-glicosidase ${ }^{6}$.

Estudos químicos anteriores descreveram a presença de $\beta$-amirina e eucaliptina, em folhas de $M$. citrifolia (Aubl.) Urb. ${ }^{7}$; flavanonas (mirciatricina I e II), acetofenas (mirciafenonas A e B) e flavonóis (mirciatricina, mearnsitrina, quercitrina, desmantina-1 e guaiajaverina), em folhas de M. multiflora ${ }^{6}$. Frações apolares de espécies deste gênero foram também investigadas. Gottlieb e colaboradores ${ }^{8}$ descreveram a composição do óleo volátil de $M$. cuprea (O. Berg) Kiaersk., caracterizando a presença de $(E)$-nerolidol. Henriques e colaboradores $^{9}$ analisaram a composição química do óleo volátil de folhas de M. acuminatissima O. Berg, M. bombycina (O. Berg) Kiaersk., M. fallax, M. glabra (O. Berg) D. Legrand e M. multiflora, identificando como produtos majoritários linalol, pinenos, germacreno D, $\beta$-cariofileno, $\alpha$-bisabolol e selinenos. Zoghbi e co-

*e-mail: amelia@farmacia.ufrgs.br laboradores ${ }^{10}$ analisaram os óleos voláteis de partes aéreas de $M$. bracteata (Rich.) DC., M. cuprea e M. sylvatica (G. Mey.) DC. e relataram a presença de mirceno, $(E)$-nerolidol, $(E)$ - $\beta$-farneseno, espatulenol, $\beta$-cariofileno, germacreno $\mathrm{D}$, selin-11-em-4- $\alpha$-ol e ciscalameneno, como os componentes mais representativos para as diversas espécies.

Como parte de projeto de investigação sistemática de óleos voláteis de espécies da família Myrtaceae ${ }^{11}$, este trabalho contempla a análise química dos óleos obtidos por hidrodestilação de folhas frescas de $M$. richardiana (O. Berg) Kiaersk., M. arborescens O. Berg, M. selloi (Spreng.) N. Silveira, M. oligantha O. Berg, M. rostrata DC., M. lajeana D. Legrand, M. obtecta (O. Berg) Kiaersk., M. pubipetala Miq e M. hatschbachii D. Legrand, coletadas de populações nativas em diferentes localidades do Rio Grande do Sul.

\section{PARTE EXPERIMENTAL}

\section{Material vegetal}

Folhas de Myrcia richardiana, $M$. arborescens, M. selloi, $M$. oligantha, M. rostrata, M. lajeana, M. obtecta, M. pubipetala e $M$. hatschbachii foram coletadas de populações nativas no Rio Grande do Sul. Todas as espécies foram obtidas de um mesmo indivíduo entre os meses de novembro e janeiro, na fase estéril, com exceção de $M$. rostrata, em fase de frutificação. Os exemplares das espécies foram identificados por M. Sobral e depositados no Herbário do Departamento de Botânica da UFRGS (ICN). O local de coleta e os números de registro no herbário são apresentados na Tabela 1.

\section{Obtenção do óleo volátil}

O óleo volátil foi obtido a partir das folhas frescas, reduzidas com o auxílio de triturador mecânico e submetidas à hidrodestilação em processo contínuo com aparelho Clevenger, durante 5 h. O cálculo de rendimento foi realizado através da relação do volume de óleo volátil recolhido do aparelho de Clevenger com a massa de material vegetal utilizada na extração ${ }^{12}$. 
Tabela 1. Dados de coleta e rendimento percentual em óleo volátil de folhas das espécies de Myrcia analisadas

\begin{tabular}{llll}
\hline Espécie & Local de coleta & ICN & Rendimento (\%) \\
\hline M. arborescens & Morrinhos do Sul - RS & 8170 & 0,2 \\
$M$. hatschbachii & Muitos Capões - RS & 8132 & 0,1 \\
$M$. lajeana & Cambará do Sul - RS & 8365 & 0,3 \\
$M$. obtecta & Cambará do Sul - RS & 8346 & 0,1 \\
$M$. oligantha & Cambará do Sul - RS & 8348 & 0,1 \\
$M$ pubipetala & Dom Pedro de Alcântara - RS & 8933 & 0,1 \\
$M$. rostrata & Cambará do Sul - RS & 8366 & 0,2 \\
$M$. selloi & Caçapava do Sul - RS & 8790 & 0,5 \\
$M$. richardiana & Morrinhos do Sul - RS & 8170 & 0,1 \\
\hline
\end{tabular}

\section{Análise química}

Para a análise química, os óleos voláteis foram diluídos na razão de 2:100 (V/V) em éter etílico. As soluções obtidas foram submetidas inicialmente à cromatografia gasosa capilar equipada com detector de ionização de chamas (CG/DIC) para análise quantitativa de seus constituintes e, posteriormente, à cromatografia gasosa capilar acoplada à espectrometria de massas (CG/EM), para a análise qualitativa.

Para a análise quantitativa utilizou-se um cromatógrafo gasoso GC-17A (Shimadzu) equipado com coluna Durabond-DB5 (30 m X $0,25 \mathrm{~mm} \mathrm{X} 0,25 \mu \mathrm{m})$. A programação de temperatura foi de 60 a $300{ }^{\circ} \mathrm{C}$ a $3{ }^{\circ} \mathrm{C} / \mathrm{min}$, temperatura do injetor de $220{ }^{\circ} \mathrm{C}$ e temperatura do detector de $250^{\circ} \mathrm{C}$. Hélio foi utilizado como gás de arraste, a uma pressão de $80 \mathrm{Kpa}$ e velocidade linear de $1 \mathrm{ml} / \mathrm{min}$. A quantificação foi obtida por integração eletrônica, pela técnica de normalização.

A análise qualitativa foi realizada utilizando-se o mesmo equipamento, nas mesmas condições, porém acoplado a um espectrômetro de massas GCMS - QP5000 (Shimadzu). A ionização foi obtida pela técnica de impacto eletrônico, com energia de 70 eV. A caracterização dos constituintes foi baseada no índice de retenção linear (Índice de Kováts) calculado em relação aos tempos de retenção de uma série homóloga de $n$-alcanos e no padrão de fragmentação observado nos espectros de massas, por comparação destes com amostras autênticas ou dados obtidos na literatura ${ }^{11,13}$, com espectroteca própria (Limberg.lib) e com espectroteca NIST62.

\section{RESULTADOS E DISCUSSÃO}

Os rendimentos dos óleos voláteis obtidos variaram entre 0,1 e $0,5 \%$ (Tabela 1). Foram identificados 67 constituintes, representando de 90,0 a $99,9 \%$ do conteúdo total dos óleos voláteis. A quantidade relativa de cada constituinte está apresentada na Tabela 2.

Todas espécies demonstraram predominância de sesquiterpenos, principalmente da série cíclica. A fração monoterpênica foi melhor representada em $M$. obtecta $(16,2 \%)$, com predominância de pinenos $(8,0 \%)$.

As principais vias de ciclização observadas, classificadas conforme descrito em Limberger e colaboradores ${ }^{14}$, foram as do cadinano (de 7,2 a 63,1\%), germacrano (de 4,6 a 64,3\%) e cariofilano (de 3,9 a $39,9 \%$ ), como ilustrado na Figura 1. A rota do bisabolano foi marcante apenas em $M$. obtecta $(34,0 \%)$, caracterizada pela presença de ar-curcumeno (19,0\%), e a série acíclica, em M. lajeana $(32,1 \%)$, com predominância de acetato de (E)-nerolidila (25,3\%). A fração monoterpênica foi mais expressiva em $M$. obtecta $(16,2 \%)$, sendo $\alpha$ pineno $(7,2 \%)$ o produto majoritário.

Das substâncias do grupo cadinano, os principais constituintes

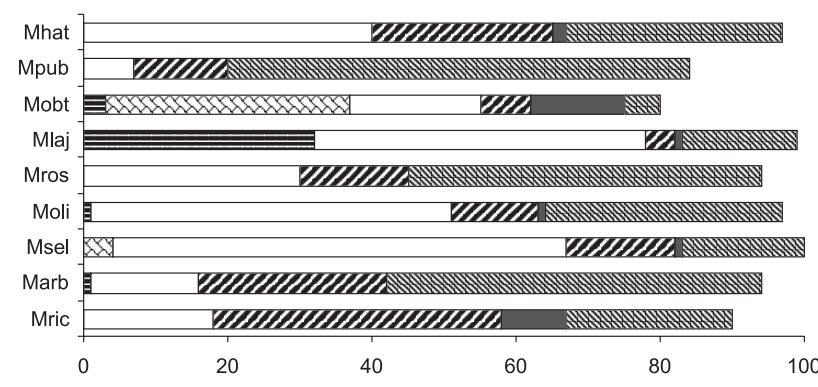

$\Xi$ Acíclico $₫$ Bisabolano $\square$ Cadinano $\square$ Cariofilano $\square$ Humulano Germacra

Figura 1. Distribuição dos constituintes dos óleos voláteis de espécies de Myrcia de acordo com a via de ciclização de sesquiterpenos. Mric $=M$. richardiana; Marb = M. arborescens; Msel $=$ M. selloi; $M$. oli $=M$. oligantha; Mros = M. rostrata $;$ Mlaj = M. lajeana $;$ Mobt = M. obtecta $;$ Mpub $=$ M. pubipetala; Mhat $=$ M. hatschbachii

identificados foram germacreno $\mathrm{D}(23,4 \%$ em $M$. lajeana $), \delta$-cadineno (17,9\% em M. oligantha) e $\alpha$-cadinol (17,2\% em M. selloi). Para o grupo germacrano, os constituintes majoritários observados foram biciclogermacreno (25,2\% em M. pubipetala), espatulenol $(32,0 \%$ em $M$. pubipetala) e globulol (15,9\% em $M$. arborescens), e para o grupo cariofilano, $\beta$-cariofileno (23,3\% em $M$. hatschbachii) e óxido de cariofileno (26,3\% em $M$. arborescens).

Algumas destas substâncias apresentam atividades biológicas, como $\beta$-cariofileno, com atividade espasmolítica ${ }^{15}$, anestésica local ${ }^{16}$ e antinflamatória ${ }^{17}$; globulol, com propriedade fungistática ${ }^{18} \mathrm{e}$ espatulenol, com propriedades antibacterianas e moderada atividade citotóxica contra células do tipo $\mathrm{KB}^{19}$.

\section{CONCLUSÕES}

Todas espécies analisadas apresentaram predominância de sesquiterpenos cíclicos, principalmente os derivados das vias de ciclização do cadinano, germacrano e cariofilano, exceto $M$. obtecta, a qual foi caracterizada por substâncias do grupo bisabolano. Estes resultados assemelham-se aos obtidos com outras espécies de Myrtaceae americanas previamente analisadas ${ }^{11,12}$, cuja predominância de sesquiterpenos cíclicos dos grupos cariofilano, germacrano e cadinano é uma constante.

\section{AGRADECIMENTOS}

O presente trabalho é subvencionado com auxílios à pesquisa FAPERGS, CAPES e CNPq. 
Tabela 2. Composição percentual do óleo volátil de folhas de espécies de Myrcia

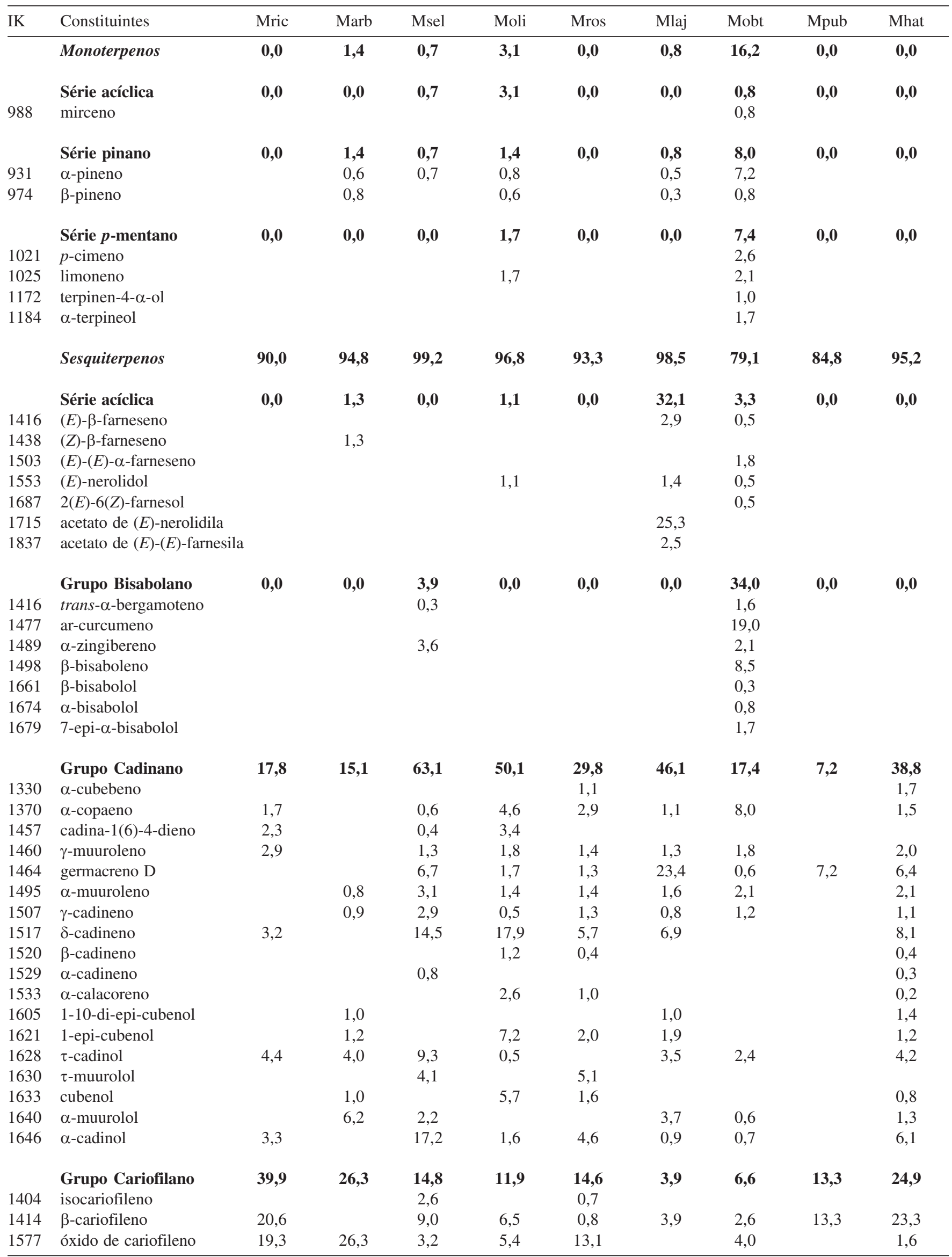


Tabela 2. continuação

\begin{tabular}{|c|c|c|c|c|c|c|c|c|c|c|}
\hline IK & Constituintes & Mric & Marb & Msel & Moli & Mros & Mlaj & Mobt & Mpub & Mhat \\
\hline & Grupo Humulano & 9,0 & $\mathbf{0 , 0}$ & 0,6 & 0,7 & $\mathbf{0 , 0}$ & 0,6 & 13,2 & $\mathbf{0 , 0}$ & 2,3 \\
\hline 1435 & $\alpha$-humuleno & 5,1 & & 0,6 & 0,7 & & 0,6 & 6,2 & & 1,5 \\
\hline 1596 & óxido de humuleno I & 3,9 & & & & & & 0,7 & & 0,8 \\
\hline \multirow[t]{2}{*}{1602} & óxido de humuleno II & & & & & & & 6,3 & & \\
\hline & Grupo Germacrano & 23,3 & 52,1 & 16,8 & 33,0 & 48,9 & 15,8 & 4,6 & 64,3 & 29,2 \\
\hline 1333 & $\delta$-elemeno & & & & & & 3,5 & & & 0,8 \\
\hline 1381 & $\beta$-bourboneno & & & 0,9 & & & & & & 1,0 \\
\hline 1403 & $\beta$-elemeno & 2,0 & & & 3,6 & & 1,6 & & & \\
\hline 1426 & aromadendreno & 2,9 & 3,1 & 0,2 & 1,7 & 3,1 & & 0,6 & 0,3 & 0,5 \\
\hline 1434 & desidroaromadendrano & & 2,5 & & & & & & & \\
\hline 1443 & allo-aromadendreno & 2,1 & 1,4 & 1,7 & 3,0 & 2,3 & & 1,3 & & 1,7 \\
\hline 1471 & $\beta$-selineno & & & & 2,4 & & 1,2 & & & 0,6 \\
\hline 1478 & viridifloreno & & & & & & & & & 1,2 \\
\hline 1481 & $\alpha$-selineno & & & & 1,3 & & 2,8 & & & 0,3 \\
\hline 1489 & biciclogermacreno & 5,7 & & 10,2 & 8,3 & 6,8 & & & 25,2 & 6,9 \\
\hline 1547 & germacreno B & & 4,9 & & 1,4 & & 3,7 & & & 0,4 \\
\hline 1560 & ledol & & 4,2 & & & 3,0 & & & & 0,3 \\
\hline 1572 & espatulenol & 3,1 & 8,9 & 1,5 & 10,2 & 17,3 & & & 31,7 & 2,6 \\
\hline 1577 & globulol & 3,6 & 15,9 & 1,7 & 1,1 & 4,4 & 1,5 & & 4,1 & 4,2 \\
\hline 1588 & epi-globulol & 3,9 & 3,0 & 0,6 & & 2,2 & & & 3,0 & 6,1 \\
\hline 1583 & 5-epi-7-epi- $\alpha$-eudesmol & & 5,9 & & & 3,9 & & 0,6 & & 1,5 \\
\hline 1602 & eudesmol* & & 1,4 & & & 1,6 & & & & 1,1 \\
\hline 1618 & 10-epi- $\gamma$-eudesmol & & & & & & 1,5 & 2,1 & & \\
\hline 1620 & isoespatulenol & & 0,9 & & & 3,8 & & & & \\
\hline \multirow[t]{2}{*}{1642} & $\beta$-eudesmol & & & & & 0,5 & & & & \\
\hline & Outros & $\mathbf{0 , 0}$ & $\mathbf{0 , 0}$ & 0,0 & $\mathbf{0 , 0}$ & $\mathbf{0 , 0}$ & $\mathbf{0 , 0}$ & $\mathbf{0 , 0}$ & 14,9 & 2,7 \\
\hline 2099 & n-heneicosano & & & & & & & & 14,9 & \\
\hline \multirow[t]{2}{*}{2100} & nonadecanal & & & & & & & & & 2,7 \\
\hline & Total & 90,0 & 96,2 & 99,9 & 99,9 & 93,3 & 99,3 & 95,3 & 99,7 & 97,9 \\
\hline
\end{tabular}

IK: Índice de retenção linear em coluna DB5. * isômero não identificado. Mric = M. richardiana; Marb = M. arborescens; Msel = M. selloii; M. oli = M. oligantha , Mros = M. rostrata $;$ Mlaj = M. lajeana , Mobt = M. obtecta $;$ Mpub = M. pubipetala Mhat = M. hatschbachii .

\section{REFERÊNCIAS}

1. Cronquist, A.; An Integrated System of Classification of Flowering Plants, Columbia University: New York, 1981; Barroso, G. M.; Sistemática de angiospermas do Brasil, Imprensa da Universidade Federal de Viçosa: Viçosa, 1991, vol. 2.

2. Marchiori, J. N. C.; Sobral, M.; Dendrologia das angiospermas-Myrtales, Ed. da UFSM: Santa Maria, 1997.

3. Corrêa, P.; Dicionário das plantas úteis do Brasil e das exóticas cultivadas, Imprensa Nacional, Ministério da Agricultura: Rio de Janeiro, 1984, vol. 2; Schultz, A.; Introdução à Botânica Sistemática, Editora da UFRGS: Porto Alegre, 1985, vol. 2; Ferro, E.; Schimini, A.; Maldonado, M.; Rosner, J.; Schmeda-Hirschmann, G.; J. Ethnopharmacol. 1988, 24, 321; Lorca, G. G.; Amat, A. G.; Gonzalez, C.; Acta Pharm. Bonaerense 1995, 14, 81.

4. Brito, N. R.; Lanetti, R. A.; A saúde pelas plantas e ervas do mundo inteiro, Ed. Ouro: Rio de Janeiro, 1996.

5. Hecht, S. M.; US pat. 280086 1981; Hecht, S. M.; US pat. 44514591984.

6. Yoshikawa, M.; Shimada, H.; Nishida, N. Li, Y., Toguchida, I.; Yamahara, J.; Matsuda, H. Chem. Pharm. Bull. 1998, 46, 113.

7. Gottlieb, O. R.; Silva, M. L.; Maia, J. G. S.; Phytochemistry 1972, 11, 1185

8. Gottlieb, O. R.; Kotesu, M. L.; Magalhães, M.; Guilherme, M.; Mendes, P.; Rocha, A.; Silva, M.; Wilberg, V.; Acta Amazônica 1981, 11, 143.

9. Henriques, A. T.; Sobral, M.; Bridi, R.; Vérin, P.; Menut, C.; Lamaty, G.; Bessière, J. M.; J. Essent. Oil Res. 1997, 9, 13.

10. Zoghbi, M. G. B.; Andrade, E. H. A.; Silva, M. H.; Carreira, L. M. M.; Maia, J. G. S.; Flavour Fragr. J. 2003, 18, 421.

11. Henriques, A. T .; Sobral, M.; Cauduro, A. D.; Shapoval, E. E. S.; Bassani, V. L.; J. Essent. Oil Res. 1993, 5, 501; Limberger, R. P.; Apel, M. A.; Menut, C.; Moreno, P. R. H.; Sobral, M.; Henriques, A. T.; J. Essent. Oil
Res. 2001, 13, 113; Limberger, R. P.; Sobral, M.; Henriques, A. T.; J. Essent. Oil Res. 2002, 14, 302; Limberger, R. P.; Sobral, M. E.; Zuanazzi, J. A. S.; Schapoval, E. E. S.; Henriques, A. T.; Pharm. Biol. 2001, 39, 308; Limberger, R. P.; Simões-Pires, C. A.; Sobral, M.; Menut, C.; Bessière, J. M.; Henriques, A.T.; Flavour Fragr. J. 2002, 17, 341; Menut, C.; Bessiere, J. M.; Ntalani, H.; Verin, P.; Henriques, A. T.; Limberger, R.; Phytochemistry 2000, 53, 975; Apel, M. A.; Limberger, R. P.; Sobral, M.; Menut, C.; Henriques, A. T.; J. Essent. Oil Res. 2001, 13, 250.

12. Farmacopéia Brasileira; $4^{\mathrm{a} e d ., ~ A t h e n e u: ~ S a ̃ o ~ P a u l o, ~ 2001 ; ~ O M S: ~ Q u a l i t y ~}$ control methods for medicinal plant materials, Organisation Mondiale de La Sante: Geneve, 1992. Série de Informes Técnicos.

13. Jennings, W.; Shibamoto, T.; Qualitative analysis of flavor and fragrance volatiles by glass capillary gas chromatography, Academic Press: New York, 1980; Adams, R. P. Identification of essential oil components by gas chromatography/mass spectroscopy, Carol Stream: Illinois, 2001.

14. Limberger, R. P; Simões-Pires, C. A.; Sobral, M.; Menut, C.; Bessière, J. M.; Henriques, A.T.; Rev. Bras. Cienc. Farm. 2002, 38, 357.

15. Cabo, J.; Crespo, M. E.; Jimenez, J.; Zarzuelo, A.; Planta Med. 1986, 20, 213.

16. Ghelardini, C.; Galeotti, N.; Mannelli, L. D. C.; Mazzanti, G.; Bartolini, A.; II Farmaco 2001, 56, 387.

17. Martin, S.; Padilla, E.; Ocete, M. A.; Galvez, J.; Jimenez, J.; Zarzuelo, A.; Planta Med. 1993, 59, 533.

18. Aleu, J.; Hanson, J. R.; Galán, R. H.; Collado, I. G.; J. Mol. Catal. B: Enzym. 2001, 11, 329.

19. Chinou, I. B.; Roussis, V.; Perdetzoglou, D.; Loukis, A.; Planta Med. 1996, 62, 377; Pacciaroni, A. D. V.; Mongelli, H.; Espinar, L. A.; Romano, A.; Ciccia, G.; Silva, G. L.; Planta Med. 2000, 66, 720; Ulubelen, A.; Topcu, G.; Eris, C.; Sonmez, U.; Kartal, M.; Kurucu, S.; Bozok-Johansson, C.; Phytochemistry 1994, 36, 971. 